\title{
Regalrechte: Patentgebühren und die Rechtsgleichheit
}

Regalrechte sind historische Monopole der Kantone. Die Rechtsprechung lässt den Kantonen bei der Ausübung ihrer Regalrechte grosse Freiräume. Obwohl es heute als unbestritten gilt, dass die Kantone auch im Rahmen ihrer Regalrechte die Rechtsgleichheit respektieren müssen, lässt es die Rechtsprechung zu, dass auswärtige Personen gegenüber Personen mit Wohnsitz im Kanton erheblich benachteiligt werden. Dies gilt insbesondere bezüglich Patentgebühren. Der Autor setzt sich mit dieser Praxis kritisch auseinander und versucht aufzuzeigen, weshalb er die Rechtsprechung des Bundesgerichts nicht mehr für zeitgemäss hält.

I. Einleitung

II. Bisherige Lehre und Rechtsprechung

Zitiervorschlag:

RAFAEL ZÜND, Regalrechte: Patentgebühren und die Rechtsgleichheit, sui generis 2021, S. 23 


\section{Einleitung}

1 Regalrechte sind traditionelle Monopole der Kantone, welche sich von anderen Monopolen in zweierlei Hinsicht unterscheiden: Einerseits dürfen sie im Gegensatz $\mathrm{zu}$ anderen Monopolen fiskalischen Zwecken dienen und andererseits bestehen sie bereits von Bundesverfassungs wegen (Art. 94 Abs. 4 BV1) und müssen nicht erst durch kantonale Gesetzgebung errichtet werden. Es bestehen folgende historische Monopole: Das Bergregal, das Fischereiregal, das Jagdregal sowie das Salzregal. ${ }^{2}$

2 Sofern der Kanton das jeweilige Monopol beansprucht, bedarf es regelmässig einer Konzession für eine ausschliessende Nutzung. Eine weniger weit gehende Nutzung kann sodann bewilligungspflichtig erklärt werden - meistens in der Form sog. Patente. ${ }^{3}$ Möglich ist jedoch auch, dass der Kanton aufgrund seiner Monopolstellung die Nutzung eines Regals verbietet (vgl. z.B. das Gesetz über die Nutzung des Untergrundes des Kantons Appenzell Innerrhoden 4 , Art. 4 Abs. 1 Bst. b, welcher das Strahlen auf dem gesamten Kantonsgebiet untersagt). Möglich ist die Nutzung dann höchstens noch über eine Ausnahmebewilligung (vgl. z.B. Art. 4 Abs. 2 GNU/AI). Zudem kann der Kanton die Regalrechte auf die Gemeinden oder andere öffentlich-rechtliche Körperschaften wie Korporationen übertragen. ${ }^{5}$

3 In der älteren Rechtsprechung betonte das Bundesgericht bezüglich Regalrechte die «praktisch uneingeschränkte Gesetzgebungsfreiheit» der Kantone. ${ }^{6}$ Dabei ist es unter den Kantonen weit verbreitet, Personen mit Wohnsitz im jeweiligen Kanton gegenüber anderen Personen bevorzugt zu behandeln. Dies geschieht entweder dadurch, dass auswärtige Personen von einer Nutzungsart gänzlich ausgeschlossen werden oder sie massiv höhere Patentgebühren zu entrichten haben. Manchmal haben auswärtige Personen auch anderweitige Nachteile zu gewärtigen.

1 Bundesverfassung der Schweizerischen Eidgenossenschaft vom 18. April 1999 (BV; SR 101).

2 Vgl. BGE124I11 E.3.b; GIOVANNI BIAGGINI, Bundesverfassung der Schweizerischen Eidgenossenschaft, Kommentar, 2. Aufl., Zürich 2017, Art. 27 N 31; sowie PIERRE TSCHANNEN / ULRICH ZIMMERLI / MARKUS MÜLLER, Allgemeines Verwaltungsrecht, 4. Aufl., Bern 2014, S. 469.

3 TSCHANNEN/ZIMMERLI/MÜLLER, (Fn. 2), S. 482 (tabellarische Übersicht) sowie S. $505 \mathrm{f}$

4 Gesetz über die Nutzung des Untergrundes des Kantons Appenzell Innerrhoden vom 29. April 2018 (GNU/AI; GS 685.000).

5 Das Bergregal wird z.B. vom Kanton Uri nicht selbst ausgeübt, sondern seit alters den Korporationen Uri und Ursern überlassen; vgl. dazu RAFAEL ZÜND, Einige Gedanken zum Strahlerrecht, Schweizer Strahler 3/2020, S. 32 .

6 Vgl. BGE 119 Ia390 E.11.b.

\section{Bisherige Lehre und Rechtsprechung}

In seiner ursprünglichen Rechtsprechung ging das Bun- 4 desgericht davon aus, dass die Kantone aufgrund ihrer traditionellen Herrschaft über die Regalrechte beinahe uneingeschränkt darüber verfügen können. Auch die Literatur schlug damals in diese Kerbe, hielt sie doch sogar die nicht sachlich begründete Ungleichbehandlung von ausländischen Personen und Schweizer Staatsbürgerinnen ${ }^{7}$ mit Wohnsitz im Kanton für zulässig. ${ }^{8}$

Im Jahr 1969 revidierte das Bundesgericht allmählich 5 seine Haltung. Im Entscheid BGE 95I 497 hielt es zunächst fest, dass die Regalrechte eine Verteilung beschränkter Ressourcen auf Nutzerinnen in billiger Weise ermöglichen sollen. Den Kantonen würden gestützt auf diese Monopole besonders umfassende Nutzungs- und Normsetzungsbefugnisse zustehen. Infolgedessen könnten sie darüber ähnlich frei verfügen wie in ihrer zivilrechtlichen Verwaltungstätigkeit (Finanzvermögen). Die Prüfungsbefugnis des Bundesgerichts beschränke sich demnach im Wesentlichen auf Willkür. Der Kanton könne als Inhaber des Fischereiregals die Fischereiberechtigung auf Personen beschränken, welche im Kanton Wohnsitz haben. Die Vorschrift in aArt. 60 $\mathrm{BV}$ verbiete nur die Ungleichbehandlung von Kantonsbürgerinnen und übrigen Schweizerbürgerinnen, die im Kanton wohnhaft seien. Eine Anknüpfung an den Wohnsitz sei zumindest dann zulässig, wenn ernsthafte Gründe eine solche Unterscheidung rechtfertigen würden. Weiter erwog das Bundesgericht, solche Gründe würden im vorliegenden Fall bestehen. In den letzten zehnJahren seien die Bewilligungsgesuche um mehr als das Doppelte angestiegen und der Kanton Graubünden würde eine übermässige Befischung befürchten. ${ }^{9}$ Zudem sei der Kanton berechtigt, sein Fischereiregal auch fiskalisch zu nutzen und deshalb von auswärtigen Personen mehr zu verlangen als von Personen, welche durch die ordentlichen Steuern bereits einen Beitrag erbringen würden. Der Grundsatz der Rechtsgleichheit gebiete es allerdings, dass sich auch Patentgebühren in einem gewissen Rahmen halten. Ihre Höhe dürfe insbesondere nicht dazu führen, dass nur noch finanzstarke auswärtige Personen eine Bewilligung erwerben könnten. ${ }^{10}$

ImJahr 1988 bestätigte das Bundesgericht seine revidier- 6 te Haltung. Damals ging es um eine abstrakte Normenkontrolle gegen einen Jagderlass des Kantons Wallis,

7 Im vorliegenden Text wird bewusst die weibliche Form benutzt, die männliche ist dabei jeweils mitgedacht.

8 Siehe Zusammenfassung der alten Rechtsprechung und Lehre in BGE114 Ia 8 E. 3.

9 BGE 95I 497 E. 2.

10 BGE 95I 497 E. 3. 
welcher ausländischegegenüber inländischen Personen mit Wohnsitz im Kanton Wallis benachteiligte, indem er für erstere höhere Patentgebühren stipulierte. Das Bundesgericht hob den Erlass mit der Begründung auf, er würde die Rechtsgleichheit gemäss aArt. 4 BV verletzen, da keine sachlichen Gründe für eine Ungleichbehandlung ersichtlich seien. ${ }^{11}$ Damit bestätigte das Bundesgericht erneut, dass die Kantone im Rahmen ihrer Regalrechte nicht nur das Willkürverbot, sondern auch das Gebot der Rechtsgleichheit beachten müssen.

7 In einem Entscheid aus dem Jahr 1993 ging es sodann um eine abstrakte Normenkontrolle bezüglich des Jagdgesetzes des Kantons Bern. Der Beschwerdeführer brachte vor, das Gesetz verletze die Rechtsgleichheit, da es auswärtige Personen mit einer drei- bis vierfachen Patentgebühr belaste. Das Bundesgericht hielt dazu fest, da die Ausübung der Jagd aufgrund des kantonalen Jagdregals den Personen mit Wohnsitz im Kanton vorbehalten werden könne, sei es auch zulässig, von ihnen höhere Patentgebühren zu verlangen. Es liege keine Verletzung des Gleichbehandlungsgebots vor. Es sei auch nicht zu beanstanden, dass ausländische Personen ohne Wohnsitz im Kanton noch stärker belastet würden als auswärtige Personen mit dem Schweizer Bürgerrecht, da letztere als Steuerzahlende bereits die Lasten des Bundesstaates mitzutragen helfen. Regalgebühren würden darüber hinaus nicht dem Kostendeckungsprinzip unterliegen, da sie auch einen Gewinn erzielen dürften und das Äquivalenzprinzip sei höchstens bedingt anwendbar. ${ }^{12}$

8 In einem weiteren Urteil aus demselben Jahr ging es um das Bergregalgesetz des Kantons Nidwalden. Die NAGRA führte dagegen staatsrechtliche Beschwerde, weil das Gesetz den Bau von Lagerstätten für radioaktive Abfälle zum Schutz von Mineralien einer Konzessionspflicht unterstellte. Entgegen seinem fünf Jahre vorher gefällten Entscheid BGE 95 I 497 betonte das Bundesgericht in diesem Entscheid die Autonomie der Kantone bezüglich ihrer Regalrechte wieder stärker und unterstrich die beinahe unbeschränkte Gesetzgebungsfreiheit. ${ }^{13}$

9 In einem Bundesgerichtsentscheid aus dem Jahr 2003 ging es um die abstrakte Normenkontrolle gegen die $\mathrm{Fi}$ schereiverordnung des Kantons Appenzell Innerrhoden. Das Bundesgericht verneinte eine Verletzung der Rechtsgleichheit gemäss Art. 8 Abs. 1 BV. Das Bundesgericht hielt es für zulässig, für Schweizer Staatsangehörige lediglich einen drei Monate andauernden Wohnsitz im Kanton zu fordern, während von ausländischen Personen zusätzlich der Besitz der Niederlassungsbewilli-

11 Vgl. zum Ganzen BGE114 Ia 8 E.3.

12 BGE119 Ia123 E.3.

13 BGE 119Ia 390 E. $11 b$. gung gefordert wird - was faktisch zu einer fünfährigen Wartefrist führen kann. Als Träger des Fischereiregals dürfe der Kanton das Fischereisystem (Pacht- oder Patentsystem) und die Voraussetzungen für die Berechtigungen grundsätzlich frei festlegen, wobei er über ein weites Ermessen verfüge. Ausgehend von der Überlegung, dass die Nutzung der lokalen Bestände in erster Linie Kantonsangehörigen und Steuerzahlenden des betreffenden Gemeinwesens zustehen solle, dürfe ein Kanton von auswärtigen Personen höhere Patentgebühren verlangen oder diese gänzlich von der Nutzung ausschliessen. Zwischen Kantonseinwohnerinnen mit kantonalem Bürgerrecht und den übrigen im Kanton wohnhaften Schweizerbürgerinnen dürfe nicht unterschieden werden (Art. 37 Abs. 2 BV), weshalb nur der Wohnsitz massgebender Anknüpfungspunkt sein könne. Ausländische Personen ohne Wohnsitz im Kanton könnten sich nicht auf Art. 37 Abs. 2 BV berufen und die Rechtsgleichheit sei nicht verletzt, da der Beschwerdeführer als ausländische Person seinen Wohnsitz nicht im Kanton Appenzell Innerrhoden habe. ${ }^{14}$

Im Jahr 2008 verneinte das Verwaltungsgericht des Kan- 10 tons Graubünden zudem eine Verletzung der Rechtsgleichheit in einem Fall bezüglich einer Zusatzbewilligung für das Strahlen. Das Gesuch des Beschwerdeführers für das Sprengen unter Verwendung von Bohrmaschinen wurde abgelehnt. Die Gemeinde als Beschwerdegegnerin machte geltend, eine solche Zusatzbewilligung werde gemäss kommunaler Gesetzgebung nur in der Gemeinde wohnhaften Personen erteilt. Mit Verweis auf BGE 44 I 170 erwog das Verwaltungsgericht, dass die Autonomie der Kantone die Befugnis in sich schliesse, von den Regalrechten in beliebigem Umfang Gebrauch zu machen. Weiter hält es mit Verweis auf BGE 119 Ia 123 fest, auswärtige Personen dürften infolgedessen gänzlich von der Nutzung ausgeschlossen werden oder es dürften ihnen wesentlich höhere Patentgebühren auferlegt werden. Der Ausschluss von auswärtigen Personen sei ein geeignetes Mittel, um der Gefahr der Übernutzung zu begegnen, und liege im gesetzlichen Gestaltungsspielraum der Gemeinde. ${ }^{15}$

\section{Kritische Würdigung}

Unbestritten erscheint heute, dass die Kantone auch im 11 Rahmen ihrer Regalrechte die verfassungsmässigen Grundrechte - und damit die Rechtsgleichheit gemäss Art. 8 Abs. 1 BV - einzuhalten haben. Während die ursprüngliche Rechtsprechung und Lehre selbst über

14 Urteil des Bundesgerichts 2P_142/2003 vom 7. November 2003 E. 3.

15 Urteil des Verwaltungsgerichts des Kantons Graubünden U 0832 vom 3.Juli 2008. 
offensichtliche Verletzungen der Rechtsgleichheit hinwegzuschauen schien, prüfte das Bundesgericht in seiner neueren Rechtsprechung zumindest, ob sich die Ungleichbehandlung in einem gewissen Rahmen hält und sie sich nicht jeglicher vernünftigen Begründung entzieht. Trotzdem legte das Bundesgericht auch in seinen neusten Entscheiden eine erstaunliche Zurückhaltung an den Tag - wobei es festzuhalten gilt, dass selbst der Neuste der hier zitierten Entscheide fast 20Jahre zurückliegt.

Seine Zurückhaltung begründet das Bundesgericht sinngemäss damit, dass den Kantonen im Rahmen ihrer Regalrechte ein grosses Ermessen zustehe und sie eine ähnliche Freiheit wie in ihrer zivilrechtlichen Tätigkeit bezüglich des Finanzvermögens geniessen würden. Dem gilt es entgegenzuhalten, dass die Kantone zwar ein gewisses Ermessen geniessen, sie aber bei den Regalrechten trotzdem nicht in ihrer privatrechtlichen Tätigkeit betroffen sind und deshalb grundsätzlich grundrechtsgebunden agieren müssen.

13 Das Bundesgericht scheint in seiner bisherigen Rechtsprechung insbesondere zu verkennen, dass nicht nur die ungleiche Behandlung bei Patentgebühren als solche, sondern auch die Höhe der Differenz gerechtfertigt sein muss. Diesen Umstand hat das Bundesgericht bisher jedoch stets ausser Acht gelassen. Ein sachlicher Grund für die unterschiedliche Höhe von Patentgebühren könnte derjenige sein, dass Kantonseinwohnerinnen bereits im Rahmen ihrer ordentlichen Besteuerung einen Teil der öffentlichen Regallasten tragen. Gemessen am gesamten Steuerhaushalt dürfte dieser Anteil jedoch äusserst gering ausfallen und pro Kantons- oder Gemeindeeinwohnerin im ein- oder tiefen zweistelligen Bereich liegen. Damit rechtfertigt sich ein Vielfaches der einfachen Patentgebühr für auswärtige Personen keineswegs. Genauso wenig können solche ungleichen Patentgebühren m.E. mit dem Argument begründet werden, der Kanton habe sogar das Recht, auswärtige Personen von der Nutzung auszuschliessen - zumal ich einen solchen Ausschluss in der heutigen Zeit als nicht mehr zulässig erachte. Ein Ausschluss von auswärtigen Personen entspricht nicht einer Verteilung in billiger Weise und lässt sich sachlich nicht mehr rechtfertigen, wie nachfolgend aufzuzeigen sein wird.

Bereits im Jahr 1969 haben Beschwerdeführende das Argument vorgebracht, die Sportfischerei habe längst die Kantonsgrenzen gesprengt. Auch auswärtige Fischer würden finanziell zum kantonalen Fischereiregal beitragen, weshalb man den Fischbestand nicht mehr als ein Gut erachten könne, das den Kantonseinwohnerinnen alleine gehöre. Das Bundesgericht ging darauf kaum ein. Es hielt lediglich fest, dafür wären den Kantonen die
Regalrechte zu entziehen, was nur durch eine Änderung der Gesetzgebung bzw. der Verfassung möglich sei. ${ }^{16}$

Was bereits im Jahr 1969 der Realität entsprach, gilt heu- 15 te - mehr als 50Jahre später - a fortiori: Die zunehmende Mobilität, das dichtere Strassennetz und die bessere Erschliessung durch den öffentlichen Verkehr führen dazu, dass die zeitlichen Distanzen zwischen Kantonen stetig abnehmen. Auch aus finanzieller Sicht verschwimmen die Unterschiede zwischen Kantonseinwohnerinnen und auswärtigen Personen zunehmend. Kantonale Regalrechte profitieren nicht nur vom nationalen Finanzausgleich ${ }^{17}$ sondern auch von Finanzhilfen des Bundes (vgl. z.B. Art.12 des Bundesgesetzes über die Fischerei18). Unter diesen Vorzeichen drängt sich eine annähernde Gleichbehandlung von Kantonseinwohnerinnen und auswärtigen Personen immer stärker auf. Insbesondere kann dem Bundesgericht nicht gefolgt werden, insofern es argumentiert, die zunehmende Mobilität spräche sowohl für als auch gegen eine stärkere Gleichbehandlung. Es mag zwar sein, dass die zunehmende Mobilität die Regalien stärker belastet. Dieser Umstand spricht jedoch in erster Linie für Nutzungsbeschränkungen, welche sämtliche Nutzerinnen rechtsgleich treffen.

Besonders stossend erscheint die heutige Rechtspre- 16 chung bezüglich des Bergregals - Ähnliches dürfte jedoch auch für das Fischerei- und das Jagdregal gelten. Im Sinne der heutigen Rechtsprechung wäre es zulässig, dass sämtliche Bergkantone das Strahlen nur noch Kantonseinwohnerinnen gestatten. Dadurch wäre es Einwohnerinnen anderer Kantone nicht mehr möglich, dieses Hobby in der Schweiz auszuüben. Dies erscheint insbesondere deshalb stossend, weil Bergkantone und damit auch ihre Regalien in besonderem Masse vom kantonalen Finanzausgleich profitieren. Trotzdem wäre es z.B. Zürcherinnen, welche einen massgeblichen Teil an die kantonalen Bergregale beitragen, nicht mehr gestattet, diese zu nutzen.

Auch bezüglich der massiven Ungleichbehandlung bei 17 den Patentgebühren erscheint die heutige Rechtsprechung veraltet. Während viele Urner und Bündner Strahlerinnen in den kristallinen Gebieten der Kantone Wallis und Bern von der Gebührenlosigkeit profitieren und rechtsgleich behandelt werden, haben Walliser und Berner Strahlerinnen in den Kantonen Uri und Graubünden teilweise massiv übersetzte Gebühren zu entrichten.

\footnotetext{
16 BGE 95I 497 E. 6.

17 Zum Finanzausgleich und zur Unterstützung der Bergkantone durch den Lastenausgleich insbesondere vgl. www.efd.admin.ch unter Finanzpolitik und Nationaler Finanzausgleich.

18 Bundesgesetz über die Fischerei vom 21.Juni 1991 (BGF; SR 923.0).
} 
Die Korporation Ursern verlangt z.B. für auswärtige Schweizerbürgerinnen mehr als das Dreifache der einfachen Patentgebühr (vgl. Verordnung über die Gewinnung von Mineralien der Korporation Ursern ${ }^{\mathbf{1 9}}$, Art. 6 Bst. a). Dabei gilt es unter Strahlerinnen als offenes Geheimnis, dass die Korporation Ursern dies aus protektionistischen Gründen tut. Ein Schutz des Mineralienbestandes wäre auch mit Massnahmen zu erreichen, welche die Rechtsgleichheit wesentlich besser respektieren.

Entgegen der Auffassung des Bundesgerichts im Jahr 1969 erheischt eine Praxisänderung hin zu einer rechtsgleicheren Behandlung von in- und auswärtigen Personen keine Gesetzes- oder Bundesverfassungsänderung. Weder die Gesetze noch Art. 94 Abs. 4 BV bestimmen die genaue Tragweite der Regalrechte. Eine solche Praxisänderung entspräche schlicht einer geltungszeitlichen Interpretation im Rahmen der teleologischen Auslegung. Auch das historische Auslegungselement dürfte dem nicht entgegenstehen. Erstens darf bezweifelt werden, dass der Verfassungsgeber die Regalrechte faktisch weitgehend dem Gebot der Rechtsgleichheit entziehen wollte, zweitens hätte ein solcher Wille keinen Niederschlag im Wortlaut von Art. 94 Abs. 4 BV gefunden und drittens sind seit Erlass der neuen Bundesverfassung über 20Jahre vergangen. Dementsprechend nimmt die Relevanz des historischen Auslegungselements ab. ${ }^{20}$ Darüber hinaus hielt es das Bundesgericht selbst unter der alten Bundesverfassung scheinbar für zulässig, eine Praxisänderung hin zu mehr Rechtsgleichheit vorzunehmen. ${ }^{21}$ Entgegen der Auffassung des Bundesgerichts lässt sich aus der Tatsache, dass die Kantone ihre Regalien fiskalisch nutzen dürfen, nicht ableiten, dass sie dabei rechtsungleich vorgehen dürfen. In diesem Sinne entspräche eine Praxisänderung m.E. einer besseren Erkenntnis der ratio legis.

19 Verordnung über die Gewinnung von Mineralien der Korporation Ursern vom 17. Mai 2015 (Strahlerverordnung; GS1320).

20 Zur geltungszeitlichen Auslegung vgl. etwa das Urteil zur staatsrechtlichen Beschwerde bezüglich Frauenstimmrecht im Kanton Appenzell Innerrhoden, BGE116 Ia 359 E. 5.c.

21 Vgl. BGE114 Ia 8 E.3.
Fraglich ist schliesslich, wie die zulässige Differenz zwi- 19 schen Patentgebühren für in- und auswärtige Personen im Rahmen einer Praxisänderung zu bestimmen wäre. Die moderne Rechtsprechung bejaht ein offensichtliches Leistungsmissverhältnis beim strafrechtlichen Wucher gemäss Art. 157 StGB22 und bei der zivilrechtlichen Übervorteilung gemäss Art. 21 OR ${ }^{23}$ grundsätzlich dann, wenn die Differenz zwischen Marktwert und der angebotenen Leistung in einem nicht reglementierten Bereich $35 \%$ übersteigt. ${ }^{24}$ Fällt die Differenz zwischen den Patentgebühren für in- und auswärtige Personen höher aus als $35 \%$, kann alleine wegen diesem Umstand noch nicht davon ausgegangen werden, dass der Marktwert der Leistung um $35 \%$ überschritten wurde - schliesslich könnte es auch sein, dass ein Kanton sämtliche Patente unter dem Marktwert verkauft. Trotzdem rechtfertigt sich m.E. die analoge Anwendung dieses Prozentsatzes auf die Regalgebühren. Damit wird ein offensichtliches Missverhältnis zwischen verschiedenen Patentgebühren verhindert und den Kantonen trotzdem noch ein Regelungsspielraum überlassen. Eine höhere Differenz zwischen Patentgebühren für in- und auswärtige Personen dürfte sich sachlich nie rechtfertigen lassen. Befürchtet der Kanton, dass ein Regal übernutzt wird, kann er auf andere Instrumente zurückgreifen, welche die Rechtsgleichheit respektieren. Er kann Beschränkungen bezüglich der Nutzungsintensität erlassen, welche sämtliche Nutzerinnen rechtsgleich treffen (Beschränkung der Anzahl Fische, welche gefischt werden dürfen; Beschränkung der Tiere, welche zum Abschuss freigegeben werden bzw. Beschränkung der Anzahl Klüfte, welche eine Strahlerin in einem bestimmten Gebiet markieren darf; Beschränkung der Patentdauer für alle Nutzerinnen und als Ultima Ratio besteht schliesslich die Möglichkeit des allgemein gültigen Verbots).

22 Schweizerisches Strafgesetzbuch vom 21. Dezember 1937 (StGB SR311.0).

23 Bundesgesetz vom 30. März 1911 betreffend die Ergänzung des Schweizerischen Zivilgesetzbuches (Fünfter Teil: Obligationenrecht; OR; SR 220).

24 Urteil des Bundesgerichts 6B_918/2018 vom 24. April 2019 E. 2.4.1 sowie 2.4.3. 